\title{
ON THE USE OF FAST BLUE, FLUORO-GOLD AND DIAMIDINO YELLOW FOR RETROGRADE TRACING AFTER PERIPHERAL NERVE INJURY: UPTAKE, FADING, DYE INTERACTIONS, AND TOXICITY
}

Anna Puigdellívol-Sánchez ${ }^{1}$, Antoni Valero-Cabré2 ${ }^{2}$ Alberto Prats-Galino ${ }^{1}$, Xavier Navarro², Carl Molander 3,4 .

${ }^{1}$ Department of Morphological Sciences, Faculty of Medicine, University of Barcelona, 08036 Barcelona, Spain.

2 Department of Cell Biology, Physiology and Immunology, Autonomous University of Barcelona, 08193 Bellaterra, Spain.

3 Department of Neuroscience, Karolinska Institutet, S-171 77 Stockholm, Sweden.

4 Department of Rehabilitation Medicine, Danderyds Hospital, S-182 88 Danderyd, Sweden.

Author for correspondence: Alberto Prats-Galino. Dept. of Morphological Sciences. Faculty of Medicine. University of Barcelona, c/ Casanova nº 143, 08036 Barcelona, Spain. Tel.: 34-934021905, Fax.:34-93-4035263, aprats@medicina.ub.es

Number of text pages (excluding title page and abstract page): 18

Number of tables: 3

Number of figures: 3

Running headline: Fluorescent tracers and peripheral nerve injury

Key words: fluorescent dyes, neurotoxicity, nerve regeneration, electrophysiology, dorsal root ganglia, motoneurones, sciatic nerve, adult rat. 


\section{ABSTRACT}

The usefulness of three retrograde fluorescent dyes for tracing injured peripheral axons was investigated. The rat sciatic was transected bilaterally and the proximal end briefly exposed to either Fast Blue (FB), Fluoro-Gold (FG) or to Diamidino Yellow (DY) on the right side, and to saline on the left side, respectively. The nerves were then resutured and allowed to regenerate.

Electrophysiological tests three months later showed similar latencies and amplitudes of evoked muscle and nerve action potentials between tracer groups. The nerves were then cut distal to the original injury and exposed to a second (different) dye. Five days later, retrogradely labelled neurones were counted in the dorsal root ganglia (DRGs) and spinal cord ventral horn. The number of neurones labelled by the first tracer was similar for all three dyes in the DRG and ventral horn except for FG, which labelled fewer motoneurones. When used as second tracer, DY labelled fewer neurones than FG and FB in some experimental situations. The total number of neurones labelled by the first and/or second tracer was reduced by about $30 \%$ compared to controls. The contributions of cell death as well as different optional tracer combinations for studies of nerve regeneration are discussed. 


\section{INTRODUCTION}

The use of various kinds of tracer substances has long been one of the fundamental methods to map connectivity in the nervous system. The characteristics of the tracers differ in several respects: uptake mechanism, fibre specificity, interaction with other tracers, method of visualization, colour, toxicity, and fading. To obtain optimal results, it is important to choose the tracer best suited for the system studied and for the specific question asked. The differences in characteristics between tracers also makes it possible to combine several dyes in the same experiment, for instance to identify neurones with dual projections by applying different retrograde tracers in the two projection areas and look for double labelled neurones (Angelov et al., 1999;

Valero-Cabré et al., 2001).

Double labelling models with different retrograde tracers have been used to study regeneration and the topographical accuracy of reinnervation after peripheral nerve injury. In one of the models used, the first tracer is applied in the peripheral target before the nerve injury to label the original population innervating the area. After nerve injury and repair, and at a time point when the regeneration process is assumed to be completed, a second tracer is applied to find the regenerating population. Theoretically, if both the first and second tracer were applied in the same target area, then the percentage of double labelled neurones would account for the degree of topographic accuracy in the regeneration process (Hendry et al., 1986; Fritzsch and Sonntag, 1991; Harsh et al., 1991; Rende et al., 1991; Madison et al., 1996). The percentage of single labelled cells with the first tracer would be representing the neuronal population that has not regenerated or has been misdirected out of its original projection area, and the single labelled cells with the second dye would indicate regenerating cells misdirected towards the area of study. Furthermore, if the second tracer is applied in an area different from the original, then double labelling would indicate axons that were misdirected from their original projecting area to another site during regeneration (Molander and Aldskogius, 1992).

Studies of the kind described above involving long-term survival of the labelled neurones are based on the assumption that the application procedure, such as injection of the tracer, and the toxic effects of the chemical dye itself do not confound the results by additional axonal damage. If this occurs, then the ability of the axons to regenerate, to take up secondary dyes at a later time point, and to conduct signals may be affected. Long term fading of the first tracer should also be minimal. The potential toxicity of tracers has been assessed in several different ways. As to FluoroGold (FG), one study suggested a toxic effect compared to True Blue after long-term accumulation in sensory neurones and spinal motoneurones (Garrett et al., 1991) whereas another study showed progressive FG fading but did not confirm toxicity (Novikova et al., 1997). Tracers like fluoro- 
green and fluoro-red were considered non-toxic because they did not cause necrosis in the injection area (Dong et al., 1996). The reduced growth of cultured-labelled cells may also be a way to detect possible labelling toxicity (Onifer et al., 1993). Thus, by using this approach, some authors have stressed that DiI is toxic in embryonic neurones (St. John, 1991) while others have not (Honig and Hume, 1989). Finally, the absence of functional alterations of labelled cells in vivo has been considered to be strong evidence for lack of toxicity, as was shown by the maintenance of homeostatic parameters after labelling of magnocellular neuroendocrine cells with Evans Blue (Weiss and Cobbett, 1992). In addition to toxic effects, the altered metabolism of the neuron after injury may change the uptake and transport mechanisms, as has been shown for horseradishperoxidase (Peyronnard et al., 1986, 1988).

We have recently suggested that sequential application of Fast Blue (FB), FG and Diamidino Yellow (DY) in peripheral innervation targets as outlined above may be useful to study topographical accuracy of sensory reinnervation (Puigdellívol et al., 1998). However, in preparatory experiments, we noted that previous injection of FB reduced subsequent uptake of DY applied by capsule in both sensory and motor axons (Puigdellívol et al., 2000). The question was then raised that the application procedure by itself, such as capsule application or injection, can result in further damage to the nerve, and that this may be tracer dependent. These considerations prompted us to extend our previous methodological studies with the aim to find out whether the application procedure and dye interactions or toxic effects affect the regenerative capacity and impulse conduction of injured neurones.

Focusing on the potential usefulness of the retrograde fluorescent tracers FB, FG and DY for studying regeneration after peripheral nerve transection and repair by sequential application as described above, the present work has been designed to assess:

1. possible differences in the number of neurones labelled by FB, FG, and DY when applied to regenerated axons three months post-injury, distal to the location of a previous nerve transection and epineurial repair. The aim is to find out whether the uptake, retrograde transport and accumulation in the soma differs among tracers after application to axons in late regeneration phase.

2. possible differences in the number of neurones labelled by FB, FG and DY three months after application to the acutely transected nerve

3. possible effects of the previous uptake and accumulation of the first tracer on the subsequent accumulation of a second tracer applied three months after the first tracer.

4. possible effects of the administration of the first tracer on the rate of neuronal survival and regeneration. By comparing the results from counting the neurones labelled at three months 
after application to the acutely transected nerve with our previous results obtained three days after application of dye immediately after injury (Puigdellívol et al., 2000), our attempt was to balance out the contribution of factors related to uptake and accumulation of dye, and to restrict the information to the contribution of fading and/or possibly cell death during the first three months.

5. whether application of dyes in a capsule on a previously transected nerve cause an additional axonal damage that can be detected by electrophysiological tests.

6. whether nerve injection with saline causes axonal damage that can be detected by electrophysiological tests.

\section{MATERIALS AND METHODS}

Thirty-two adult female Sprague Dawley rats (250-330 g) were used in the present study. All animals were obtained from Harlan Interfauna Iberica S.A., maintained in the Animal Care Service, Faculty of Medicine, University of Barcelona, and treated in compliance with the ethical guidelines of this center. Anaesthesia was initiated with ether and then continued with chloral hydrate $(300 \mathrm{mg} / \mathrm{kg})$ during all surgical procedures and perfusion. Bilateral experiments were used in order to reduce the number of animals.

\section{Labelling procedures, questions 1-5}

The sciatic nerves were exposed bilaterally at the level of the thigh and transected by means of sharp microscissors. A swab was placed underneath the cut nerve to prevent spread of leaked tracer or of saline. The sciatic nerve of the right hindlimb was used for questions 2, 3, 4 and 5, and the sciatic nerve of the left hindlimb for questions 1 and 3 (see Introduction and Table I).

The proximal end of the transected sciatic nerve on the right hand side was inserted into a plastic capsule containing $1.5 \mu \mathrm{l}$ of 5\% FB (Sigma, $n=8$ ), 10\% FG (Fluorochrome Inc., $n=8$ ) or 5\% DY (EMS-Polyloy, $n=8$ ) in distilled water and kept in this position for 30 minutes. The proximal end of the transected sciatic nerve on the left hand side was inserted into a plastic capsule containing $1.5 \mu \mathrm{l}$ of saline. After 30 minutes, the proximal stump was cleaned, reapposed and sutured to the distal stump using nylon monofilament 10-0 suture. The skin was sutured and the rats allowed to recover.

After three months to allow for nerve regeneration and peripheral reinnervation, the animals underwent electrophysiological studies (see below). Two to four days later, the sciatic nerves were bilaterally transected $10 \mathrm{~mm}$ distal to the first suture. On the right side, the proximal stump was inserted into a plastic capsule containing 5\% DY in the groups that had previously received FB or 
FG (FB-DY and FG-DY groups, respectively), or 5\% FB in the group that had previously received DY (DY-FB group). On the left side, the proximal stump was inserted into a plastic capsule containing FB, FG or DY. They were then assigned to groups S-FB, S-FG and S-DY, respectively (Table I and Figure 1). After 30 more minutes both nerves were cleaned, the skin was sutured and the animals were allowed to survive for 5 extra days before perfusion.

\section{Nerve damage by nerve injection, question 6.}

Additional rats $(n=8)$ were used to detect possible nerve damage after nerve injection. The tibial nerve was dissected at the level of the knee joint. The tip of a 26 gauge needle attached to a 10 $\mu 1$ Hamilton syringe was introduced about $4 \mathrm{~mm}$ distal to the origin of the gastrocnemius branches, pushed 1-3 $\mathrm{mm}$ in proximal direction and $1 \mu \mathrm{l}$ of saline solution slowly injected. After this procedure, the skin was sutured and the rats allowed to recover. The contralateral side was left intact. Electrophysiological evaluation was performed three months later.

\section{Electrophysiology}

Electrophysiological evaluation was performed under pentobarbital anesthesia (40 mg/kg), three months after dye application or nerve injection. During the tests, the animals were placed over a warmed flat coil controlled by a hot water circulating pump to maintain skin temperature above $32^{\circ} \mathrm{C}$. The sciatic nerve was stimulated by two needle electrodes percutaneously inserted at the sciatic notch, applying single rectangular pulses of $0.05 \mathrm{msec}$ duration up to the voltage required to obtain a maximal evoked response. Compound muscle action potentials (CMAP), evoked by stimulation of motor nerve fibres, were recorded from the medial gastrocnemius (GM), anterior tibialis (TA), and plantar (PL, from the 3rd metacarpal space) muscles using monopolar needles (Navarro et al., 1994, 1996). Compound nerve action potentials (CNAP), elicited by stimulation of large sensory nerve fibres, were recorded with small needle electrodes inserted near the digital nerves in the proximal (active electrode) and distal (reference electrode) phalanges of the second, fourth and fifth toes. Compound action potentials were amplified and displayed on the oscilloscope (Sapphyre 4ME, Vickers) to measure the amplitude of the negative peak and the latency to the onset.

\section{Fixation, sectioning, microscopic examination}

Finally, rats were anaesthetised and a thoracotomy was performed. An intracardial injection of $1000 \mathrm{UI}$ of heparin/kg body weight was followed by perfusion through the ascending aorta with $100 \mathrm{ml}$ saline and then with $500 \mathrm{ml}$ 4\% paraformaldehyde and $10 \%$ sucrose in phosphate buffer 
(PB, $\mathrm{pH}=7.40$ ) for 20 minutes. The lumbar dorsal root ganglia (DRG) L3-6 and corresponding spinal cord segments were removed and post fixed for three hours in the same fixative + sucrose solution. After that, the L4-5 DRGs were cut on a cryostat in $10 \mu \mathrm{m}$ thick longitudinal sections, and the spinal cord and L3 and L6 DRGs were cut in $30 \mu \mathrm{m}$ thick serial longitudinal sections. Sections were all thaw-mounted on chrome-alum gelatinised (5\%) slides and coverslipped using an antifading solution containing $1 \%$ paraphenylenediamine and $10 \%$ phosphate buffered saline in glycerol.

The sections were examined in an Olympus Vanox fluorescence microscope using appropriate filter combinations (ultraviolet light filters: DM 400 dichroic mirror and UG1 excitation filter, which gives $365 \mathrm{~nm}$ excitation and $420 \mathrm{~nm}$ emission wave lengths, and violet light filters: DM 455 dichroic mirror and BP 405 exciter filter which gives $405 \mathrm{~nm}$ excitation and $455 \mathrm{~nm}$ emission wavelengths). Neuronal profiles with an identifiable nucleus were counted in every tenth DRG section and in every fourth spinal cord section (see Figure 2 and Puigdellívol et al.,1998, 2000 , for more information on the identification of labelled neurones). The three tracers can be easily differentiated by the ultraviolet filter; FB is blueish, FG is reddish, and DY is pale yellow. In case of double labelling, FB and FG may hide DY if the ultraviolet filter is used. This difficulty was solved by checking the cells also by the violet filter which clearly facilitated the visualisation of the DY. The total number of labelled profiles counted in each case using every tenth section of DRGs L4-5 and every fouth section in the spinal cord, as well as means and standard deviations from total cell counts and percentages are presented in Table II and Fig 3. No corrections were made for the possibility of counting split cells twice in different sections.

\section{Statistical analysis}

Total cell counts among groups were compared by means of an ANOVA-test after verifying the normality of the variable by the Kolmogorov-Smirnov test. If different variances were detected by a Levene's test, a Kruskall-Wallis test was used. If significant differences among groups were detected, Student's t-tests were used to compare differences between the different pairs of groups of the comparison, or Mann-Whitney U test when occasional different variances were found. Paired Wilcoxon $\mathrm{W}$ tests were used to compare mean double labelling percentages between DRG and spinal cord in the same experiment, and Kruskal-Wallis and Mann-Whitney U tests to compare percentages among groups. Counts of profiles showing the tracer applied before regeneration and numbers of profiles labelled with the tracers used after the regeneration period were compared with those obtained after a control labelling of a sciatic nerve, in which the tracer was applied in a capsule and the animal was perfused after five days (Puigdellívol et al., 2000). Average amplitudes 
and latencies of CMAP and CNAP among groups were compared by means of a Kruskall-Wallis followed by Mann Whitney U test.

\section{RESULTS}

1. Differences in the uptake of $F B, F G$ and $D Y$ by regenerating, previously non-labelled sensory and motor axons.

When the different tracers were applied at the end of the regeneration period ( 90 days; groups S-FB, S-FG and S-DY) to previously non-labelled nerves, distal to the previous injury site, DY tended to label fewer cells than FB and FG in both the DRG and spinal cord ventral horn. Significant differences were found only between DY and FG $(\mathrm{p}=0.001)$ in the motoneuronal population (Table II, 1 and Fig. 3).

2. Differences in the combined contribution of cell body accumulation and fading of $F B, F G$ and DY at three months after application to the transected nerve (to label the original population before regeneration).

These results are based on observations in animals that received the first tracer just after injury, and the second tracer after regeneration. Here, only the first tracer is considered. When comparing the numbers of cell profiles containing the tracers applied before regeneration to label the original population of the nerve, no significant differences were found among the groups (FBDY, FG-DY and DY-FG) in the DRG ( $\mathrm{p}=0.8894)$. Differences were found, however, for the motoneuronal population, where significantly fewer neurones were labelled by FG after 90 days (FG-DY group), compared to FB ( $\mathrm{p}=0.001)$ and DY $(\mathrm{p}=0.0001)$ in the FB-DY and in the DY-FB groups respectively (Table II, 2 and Fig. 3).

To obtain an indirect measure of the fading of the first tracer, the percentage of cell profiles containing only the second tracer (\% regarding all single and double labelled profiles together) was calculated. It was found to be $2.4 \%, 5.5 \%$, and $19.2 \%$ in the DRGs for the FB-DY, FG-DY, and DY-FB groups respectively. Corresponding percentages in the spinal cord motor pool were $1.4 \%$, $27.2 \%$, and $8.1 \%$ respectively.

\section{Possible effects of the previous uptake and accumulation of the first tracer on regeneration} capacity of labelled neurones and on the subsequent uptake of a second tracer applied three months after the first tracer.

These results are based on observations in animals that received the first tracer just after injury, and either DY or FB as second tracer after regeneration. Here, only the second tracer is 
considered. In the DRGs, similar mean numbers of DY (second tracer) labelled profiles were found in the FB-DY and FG-DY groups, but tended to be lower compared to cases where FB was used as second tracer (DY-FB group). Between groups, comparisons in the DRG showed that the FB counts in the DY-FB group were significantly higher than the DY counts in the FG-DY group $(\mathrm{p}=0.009)$, but not compared to the DY counts in the FB-DY group ( $\mathrm{p}=0.060)$, possibly due to the high variability found in this group. For motoneurones also, significant differences were found among groups ( $\mathrm{p}=0.0076)$. The number of profiles labelled with DY in the FB-DY and in the FG-DY group were significantly lower than the FB counts in the DY-FB group $(p=0.011$ and $p=0.001$, respectively; Table II,3 and Fig. 3).

When the FB counts in the DY-FB group (Table II,3 and Fig. 3) were compared to the FB counts obtained after a single application of this tracer at the end of the regeneration period (the $S$ FB-group, Table II,1 and Fig. 3), no differences were found neither for the DRGs ( $\mathrm{p}=0.638$ ) nor for the motoneurone pool $(\mathrm{p}=0.612)$. However, corresponding counts for DY in the FB-DY and FGDY groups tended to be lower than those found after a single application of DY (S-DY-group) at 90 days postlesion. This did not reach significance in the DRG $(\mathrm{p}=0.070)$, but did so in the motoneurone pool $(\mathrm{p}=0.048)$ where the number of DY cells obtained after a single application of DY (S-DY) at 90 days was greater than the number of DY cells in the FG-DY group ( $\mathrm{p}=0.011$ ).

\section{Effects of the administration of the first tracer on axonal regeneration and neuronal survival.}

The percentage of double labelled DRG cells among the double labelled plus the cells single labelled by the first tracer was 54\% for the FB-DY group, $64 \%$ for the FG-DY group, and $82 \%$ for the DY-FB. Corresponding percentages for the motoneurones were $26 \%, 37 \%$ and $66 \%$, respectively (Table II,4). Significant differences were found among groups for both the DRGs $(\mathrm{p}=0.021)$ and motoneurones $(\mathrm{p}=0.027)$. The ratio of double labelling in the DY-FB group was greater than in both the FB-DY group ( $\mathrm{p}=0.014$ in DRG and 0.038 in the spinal cord) and the FGDY group ( $\mathrm{p}=0.022$ and $\mathrm{p}=0.014$ in DRG and spinal cord). The differences in percentage of double labelling between the DRGs and the motoneurone pool in the different groups were all statistically significant ( $\mathrm{p}=0.018, \mathrm{p}=0.028$, and $\mathrm{p}=0.028$ for FB-DY, FG-DY, and DY-FB groups, respectively).

When the mean total numbers of labelled cell profiles with either one of the two tracers in the different groups were compared (Table II,5 and Fig. 3), no differences were found in DRG among groups $(\mathrm{p}=0.346)$ but significant differences were found in the spinal cord $(\mathrm{p}=0.0015)$, where the FG-DY group showed a lower number of labelled profiles when compared to FB-DY $(\mathrm{p}=0.008)$ and DY-FB groups $(\mathrm{p}<0.0001)$. 


\section{Electrophysiological evaluation.}

Nerve conduction tests were carried out at 90 days postoperation in all groups, before the application of a second tracer. In three groups the right sciatic nerve had been exposed to FB, FG and DY dyes (FB-DY, FG-DY and DY-FB) at the time of operation while the left sciatic nerve had been exposed to saline. Finally, muscle and sensory nerve conduction tests were performed in the group used for saline injection in the right tibial nerve (INJ group) and in the contralateral intact hindlimb (CONTR). Results of the amplitude and latency of the evoked CMAP and CNAP are shown in Table III. In all the rats subjected to sciatic nerve section and repair there was evidence of reinnervation of the muscle and nerve targets evaluated, although values for the amplitudes were significantly lower and values for the latencies longer than control values.

By 90 days postoperation there were recordable CMAPs in the three muscles tested in all the experimental animals. The CMAP amplitude ranged about 59-65\% in GM and 47-58\% in TA muscles with respect to control values, while there was lower, $18-21 \%$, in the distal plantar muscles. Overall, the six groups compared had achieved a similar degree of motor reinnervation. No significant differences were found in the final recovery of GM and PL CMAP amplitude and latency between groups ( $>0.05)$. Contrarily, groups FB-DY and S-FB had significantly lower amplitude for the TA muscle than the other groups (Table III).

The CNAP amplitude ranged between $24-38 \%$ for the 2 nd digital nerve, $16-32 \%$ for the 4 th and the 5 th digital nerves. The CNAP of the 2 nd digital nerve recovered in 7/7 animals in DY-FB group (100\%), 6/8 in FG-DY group (75\%) and 5/8 in FB-DY group (62\%). In the 4th digital nerve CNAPs were recorded in 1/8 (12\%), 7/8 (87\%) and 4/7 (57\%), and in the 5th digital nerve in 4/8 $(50 \%), 7 / 8(87 \%)$ and 6/7 (85\%) rats for groups FB-DY, FG-DY and DY-FB respectively. For calculations, when no CNAP was recorded, the amplitude was given a value of zero and the latency was considered as missing value (Navarro et al., 1994).

No statistically significant differences for the CNAP amplitudes and latencies were found between the different groups in the 2 nd and 5th digital nerves tested ( $>>0.05$ ). In contrast, the FBDY group showed a lower mean CNAP amplitude for the 4th digital nerve than group FG-DY. There were no significant differences between the results of nerves with a fluorescent dye applied and the contralateral nerves with only saline applied at the time of operation for any of the nerve conduction parameters (Table III).

\section{Injection of the tibial nerve.}

In order to examine if the nerve injection of a solution, a procedure that is commonly used to inject tracers to intact nerves, has noticeable effects on the functional properties of the nerve, 
electrophysiological tests were performed three months postinjection in an extra group of animals and compared to control values obtained in the contralateral intact hindlimb. The results did not show significant differences ( $>0.05)$ between the saline injected and the intact nerves for any of the muscle (GM, TA and PL) and nerve action potentials (digital nerves).

\section{DISCUSSION}

Technical considerations

The uptake, transport and toxicity of the dyes may be influenced by the solution medium and the concentration of the tracer. In this paper we dissolved the dyes in water according to the recommendations in several previous publications (Bentivoglio, 1980; Keyzer, 1983; Schmued and Fallon, 1986) and because we had successfully used them obtaining similar numbers of labelled neurones after subcutaneous application (Puigdellívol et al, 1998). We used a higher concentration of FG $(10 \%)$ as we had found in preliminary experiments that this concentration seems to reduce the problem of fading in the DRG at 3 months after tracer injection in the toes (unpublished).

The second tracer was applied distal to the original lesion with the aim to label regenerating axons selectively. In order to reduce the possibility of proximal spread of tracer to axons that had not regenerated, we: a) used capsule application to a transected nerve instead of nerve pressure injection, b) we applied the capsule as far distal as possible (at least 10mm away from the original lesion) without exclusion of branches, c) we carefully checked by visual inspection that no tracer spread proximally along the nerve. We cannot fully rule out, however, that some of the dye was taken up also by proximal non-regenerating axons.

\section{Labelling with the first tracer: neuronal death versus tracer fading.}

In a perfect sequential double labelling model to study regeneration, one of the prerequesites is that the labelling corresponding to the first tracer, applied at the time of injury, should identify the entire original neuronal population of the nerve at the end of the follow-up period. However, our data show that the number of labelled cell profiles after 90 days regeneration period is about 20$30 \%$ lower than the number obtained in previous experiments involving double labelling of the normal sciatic nerve with DY and FB using a survival time of only three days (Puigdellívol et al., 2000). The most likely explanations for the decrease are dye fading and/or neuronal death.

Progressive fading of FG (up to a 90-95\%) in motoneurones has been previously described by using 2\% FG (Crews and Wigston, 1990; Novikova et al., 1997). Our FG-DY group showed that about $27 \%$ of the motoneurones contained only DY labelling (second tracer). Since application of a tracer in a capsule is likely to label most of the nerve fibres, it is reasonable to assume that the DY 
single labelled cells represent surviving neurones in which the FG has faded during the regeneration period rather than cell death due to the lesion per se, or to toxic effects which, however, can not be ruled out (Naumann et al. 2000). As the same counting method was used for all three tracers, the relative magnitude of the decrease should be similar even if stereological techniques were used.

Furthermore, the CMAP (for motor axons) and the CNAP (for large sensory afferents) amplitude and latency were not significantly different in the FG group compared to those obtained in the other groups. We find unlikely that normal electrophysiological parameters is consistent with extensive cell death as a result of a toxic effect of FG, and that a more likely explanation for the diminished FG counts found in this study was due to FG fading.

Some DY reduction was also demonstrated in the DY-FB group. About $19 \%$ of DRG cells and $8 \%$ of motoneurones showed only FB, the second tracer applied 90 days after DY labelling. As the labelling can be assumed to be maximal in the control situation, yielding more than $90 \%$ double labelled neurones with these two tracers (Puigdellívol et al., 2000), the existence of an increased percentage of neurones single labelled by FB (second tracer) found in this study after three months suggests that DY (first tracer) had faded. It can not be excluded that the second tracer in some cases obscured the detection of weak fluorescence from the first tracer, due to a certain degree of fading. We did not see fading of DY in neither DRGs nor the ventral horn at 60 days after injection of this tracer in the uninjured tibial nerve (Puigdellívol et al., 2000). This difference may be explained by a prolonged availability of tracer at the injection site (Innocenti et al., 1986, Rende et al., 1991) in the uninjured nerve as compared to capsule application to the transected nerve where the capsule is removed and the nerve cleaned so that little tracer will remain available for uptake. In the other groups (FB-DY in DRG and motoneurones, and FG-DY in DRG) a small percentage of cells labelled only by the second tracer applied was observed. Although this finding could indicate a limited fading of FB and FG, it could also be due to certain difficulties of DY to label regenerating neurones (see below). However, as the numbers of FB, FG and DY cells which remain visible after three months were similar, it is possible that all three tracers were subjected to a comparable degree of fading in the DRGs.

The results mentioned above indicate that using the ratio between cells labelled by the first tracer after three months and the corresponding numbers obtained in acute control experiments as a measure of cell survival results in an understimation of the number of surviving neurones due to tracer fading. An alternative approach would be to use the total number of cells labelled with any of the tracers in the different groups as the maximal detectable number of surviving cells, and to compare with the total profile counts obtained in acute control nerves (Puigdellívol et al., 2000). The reduced percentage of FG-DY labelled motoneurones (about 63\% reduction) may be explained 
by FG fading (see above) but also by tracer interactions (see below). As for the FB-DY and DY-FB groups, the reduction was about 27 to $39 \%$ in DRGs, and 30 to $32 \%$ in motoneurones as compared to controls. This reduction might also to some degree be attributed to an inability of the second tracer to label a certain population of surviving neurones, or to fading of the first tracer in nonregenerating but surviving neurones. Nevertheless, the percentages of cell loss estimated by this method are similar to the range of neuronal death in DRG after peripheral nerve injury reported in other studies (Arvidsson et al., 1986; Himes and Tessler, 1989; Ygge, 1989; Vestergaard et al., 1997; Ljungberg et al., 1999).

The problem of whether or not some motoneurones die after peripheral nerve injury has been debated for a long time. Thus, authors have reported a non-significant motoneuronal loss of about $10 \%$ after median and ulnar nerve transection in adult mice (Pollin et al., 1991), about 15\% after forelimb amputation in mice (Crews and Wigston, 1990), and about $20 \%$ after nerve section in the rat axillary region (Gu et al., 1997). A loss of about 20 to $30 \%$ hypoglossal motoneurones (Snider and Thanedar, 1989; Törnqvist and Aldskogius, 1994) and facial motoneurones (Yu, 1988; Mattson et al., 1998) was described after cranial nerve section in adult rats. Several authors found a non-significant loss of about $10 \%$ motoneurones after sciatic nerve injury (Vanden-Noven et al., 1993; Lowrie et al., 1994; Valero-Cabré et al., 2001) and no changes with respect to the contralateral side was found after section and repair of the rat femoral nerve (Al-Majed et al., 2000). These varying results may to some extent be due to counting problems, but also to age, postoperative survival time, and proximity of lesion (see Lowrie and Vrbová, 1992; Snider et al., 1992; Fu and Gordon, 1997). By analogy to findings in the DRGs (Ygge and Aldskogius, 1984; Arvidsson et al., 1986; Groves et al., 1997), an increased amount of neuronal disappearence in the motor columns could take place if very long survival times were studied. Furthermore, the degree of motoneuronal (Martin et al., 1999) and DRG neuronal (Ygge, 1989) death after axonal injury in adult mammals was found to be mainly dependent on the severity of the injury and on the distance from the axonal injury to the neuronal body.

Further studies combining tracing with histology and detection of apoptosis (Groves et al., 1997, 1999; Wada et al., 1999), and using stereological counts (Sterio et al., 1984) should be designed to assess the possibility of motoneuronal death with more certainty.

\section{Labelling after regeneration: reduced regeneration versus tracer interaction.}

When using sequential application of fluorescent tracers to evaluate axonal regeneration after nerve injury, the second tracer should account for the regenerating population. The number of neurones labelled by this tracer could be misleading, however, if regenerating neurones change their 
capacity to uptake and transport tracers as compared to normal (Peyronnard et al., 1986, 1988), or if labelling by the first tracer interferes with the uptake of the second tracer (Puigdellívol et al., 2000).

No significant differences in the numbers of labelled DRG neurones were found for the three tracers used in this study after single application to the previously transected nerve after the regeneration period, indicating that there was no major difference in the uptake, transport or accumulation capacity between tracers. The S-DY group showed the lowest number within this comparison, but not significantly different from that found in the double labelling procedure. This indicates that the reduced DY numbers in DRG in the sequential labelling situation was not attributable to interaction with the previously applied tracer. Significantly reduced DY counts were found, however, in both the single and double labelling models for motoneurones. Furthermore, DY counts in the FG-DY and FB-DY groups were reduced when compared to the S-DY group, suggesting that the previous application of both FG and FB somehow affected the subsequent labelling with DY. We are unable to say whether this is related to a selective toxic effect of the first applied tracer, to reduced uptake of DY, or possibly problems with visualization. Furthermore, we don't have an explanation for the apparently selective affection of the DY uptake in the previously FG labelled motoneurones, but not DRG neurones. Nevertheless, these findings may reduce the usefulness of those dye combinations. No significant differences where found among the profile counts of regenerating cells labelled by FB in the double and single labelling model, indicating that the previous application of DY did not interfere with FB uptake.

Double labelling as an indication of the index of regeneration.

The percentage of double labelling cells should account for the percentage of axons that have regenerated. This figure is, however, likely compromised by the factors described above, particularly fading of the first tracer and reduced uptake of the second tracer by regenerating neurones. An index of regeneration might be obtained by comparing the total number of cell profiles in a control sample to the total number of labelled profiles in the single labelling model or the total number of profiles stained with the second tracer in the double labelling model (see above).

Because the number of regenerating cells labelled by DY were lower than for the other tracer combinations, only FB and FG are considered here for this comparison. FB labelled a similar number of regenerating neurones when applied alone or in the DY-FB group (59-62\% in DRG and $45-48 \%$ in motoneurones, compared to a control sciatic nerve). Similar results were found with FG, which labelled about $55 \%$ of regenerated neurones in the single labelled group for both DRG and motoneurones. However, the percentages given should be considered with caution as stereological techniques were not used in this study. 
The percentages of labelled regenerating motoneurones obtained by single and by the double labelling procedures were reduced when compared to the percentages of regenerating DRG cells in all groups. This seems to be in agreement with the previously reported reduction in motoneuronal regeneration as compared to sensory neurones (Suzuki et al., 1998), and to the decreased amount of target reinnervation by thick motor and sensory fibres compared to thin sensory and autonomic fibers after peripheral nerve injuries (Navarro et al., 1994; Verdú and Navarro, 1997).

We have not explored the use of DY as the first tracer and of FG as the second tracer because we found that the DY-FB combination appeared to work well. Another reason was that it is more difficult to detect neurones labelled with FG and DY through the same filter. It is concluded that FB seems to be a good candidate to be used as second tracer following application of DY as primary tracer

\section{Neuronal labelling and electrophysiological recordings.}

When attempting to correlate the counts of retrogradely labelled neurones with the functional tests several points have to be taken into account. The electrophysiological tests used in the present study evaluate the conduction of impulses by large myelinated $(A \alpha \beta)$ nerve fibers and not the functional status of thin myelinated and unmyelinated fibers. Thus, the CNAP amplitudes recorded in sensory nerves, such as the digital nerves, are not directly comparable with the DRG counts which include all types of afferents. The CNAPs recorded from the nerves in the distal digits are assumed to be mainly sensory CNAPs, since it has been previously described that regenerating motor axons tend to reinnervate motor branches (see Madison et al, 1996 for references).

Regarding the motor system, the percentage of regenerating cells and the CMAP amplitude may be more comparable, since motoneurones have only myelinated axons. However, the number of regenerating motor axons can not be directly correlated with the muscle potentials recorded due to two well known phenomena; the terminal sprouting of regenerative motor axons that may increase to 2-3 times the size of their complementary motor units (Brown et al., 1981), and the changes in type of motor units after denervation and reinnervation (Gordon et al., 1999).

Nevertheless, the question we addressed was whether there is any tracer combination that may interfere the electrophysiological recordings. Occasional significant differences among groups were found for the CMAP or CNAP in a particular muscle or nerve, but the differences were not consistent for most of the amplitudes and latency values in the intergroup comparisons. It is worth to note that there were no overall differences in the conduction tests between the right sciatic nerve labelled with a fluorescent tracer at the time of injury, and the contralateral nerve to which saline had been applied. The only noticeable interference appeared in the FB-DY group, in which CNAPs 
were detected in the fourth digit in only one of the eight rats. However, this abnormally low proportion of reinnervation can be considered as incidental, since all the other parameters in this group (including CNAPs of the second and fifth digital nerves) were not different with respect to the other five injured groups.

Finally, the group of rats which received only a saline nerve injection showed no differences in the motor and sensory recordings with respect to the control side, indicating that the injection itself caused negligible changes in the nerve function and is unlikely to interfere with complementary electrophysiological studies. This does not exclude that a mild injury to the nerve fibers was inflicted.

Use of FB, FG or DY in regeneration studies.

In conclusion, the results of the present study indicate that FB, FG and DY might be used to study nerve regeneration in single or double labelling models with some limitations: the considerable FG fading in motoneurones and the reduced labelling efficacy of DY in regenerating cells (especially when applied as a second tracer), should be taken into account. Furthermore, the difficulties in visualizing double DY-FG cells through the same filter (Puigdellívol et al., 1998) makes the DY-FB combination more suitable for a double labelling model. In that case, a certain degree of DY fading is to be accounted for which means that only a sample of the original population will be studied. The tracers may be applied by means of a nerve injection with negligible damage to the nerve. This morphological approach may be combined with electrophysiological tests. Either FB and FG appear to be useful if the use of a single tracer applied after the regeneration period is desired.

\section{Acknowledgments}

We want to thank Dolors Fuster for efficient technical assistance. This work was supported by grant from the Secretaría de Estado de Universidades e Investigación, Grant Number PM99/0173, the Fundació Marató TV3 (003/97), the FIS (00-031-2) from Spain, and the Swedish Medical Research Council, project No.11566 and 13124. 


\section{REFERENCES}

Al-Majed AA, Neumann CM, Brushart TM, Gordon T. Brief electrical stimulation promotes the speed and accuracy of motor axonal regeneration. J Neurosci 2000;20:2602-8.

Angelov DN, Skouras E, Guntinas-Lichius O, Streppel M, Popratiloff A, Walther M, Klein J, Stennert E, Neiss WF. Contralateral trigeminal nerve lesion reduces polyneuronal muscle innervation after facial nerve repair in rats. Eur J Neurosci 1999;11:101-10.

Arvidsson J, Ygge J, Grant G. Cell loss in lumbar dorsal root ganglia and transganglionic degeneration after sciatic nerve resection in the rat. Brain Res 1986;373:15-21.

Bentivoglio M, Kuypers HGJM, Castsman-Berrevoets CE, Loewe H, Dann O. Two new fluorescent tretrograde neuronal tracers which are transported over long distances. Neurosci Lett 1980;18:25-30.

Brown MC, Holland RL, Hopkins WG. Motor nerve sprouting. Annu Rev Neurosci 1981;4:17-42.

Crews LL, Wigston D. The dependence of motoneurons on their target muscle during postnatal development of the mouse. J Neurosci 1990;10:1643-53.

Dong K, Qu T, Ahmed FA, Zhang L, Yamada K, Guison NG, Miller M, Yamadori T. Fluoro-Green and Fluoro-Red: two new fluorescent retrograde tracers with a number of unique properties. Brain Res 1996;736:61-7.

Fritzsch B, Sonntag R. Sequential double labelling with different fluorescent dyes coupled to dextran amines as a tool to estimate the accuracy of tracer application and of regeneration. $\mathbf{J}$ Neurosci Meth 1991;39:9-17.

Fu SY, Gordon T. The cellular and molecular basis of peripheral nerve regeneration. Mol Neurobiol 1997; 14:67-116.

Garrett TW, McBride RL, Williams JK, Feringa ER. Fluoro-Gold's toxicity makes it inferior to True Blue for long-term studies of dorsal root ganglion neurones and motoneurons. Neurosci Lett 1991;128:137-9.

Gordon T, Tyreman N, Rafuse VF, Munson JB. Limited plasticity of adult motor units conserves recruitment order and rate coding. Progr Brain Res 1999;123:191-202.

Groves MJ, An SF, Giometto B, Scaravilli F. Inhibition of sensory neuron apoptosis and prevention of loss by NT-3 administration following axotomy. Exp Neurol 1999;155:284-94.

Groves MJ, Christopherson T, Giometto B, Scavarilli F. Axotomy-induced apoptosis in adult rat primary sensory neurons. J Neurocytol 1997;26:615-24.

$\mathrm{Gu} \mathrm{Y}, \mathrm{Zvonko} \mathrm{S}, \mathrm{Wu} \mathrm{W}$. The effects of remaining axons on motoneuron survival and NOS expression following axotomy in the adult rat. Dev Neurosci 1997;19:255-9. 
Harsh C, Archibald SJ, Madison RD. Double-labelling of saphenous nerve neurone pools: a model for determining the accuracy of axon regeneration at the single neuron level. J Neurosci Meth 1991;39:123-9.

Hendry LA, Hill CE and Watters DJ. Long-term retention of Fast Blue in sympathetic neurones after axotomy and regeneration-demonstration of incorrect reconnections. Brain Res 1986;376:292-8.

Himes BT, Tessler A. Death of some dorsal root ganglion neurons and plasticity of others following sciatic nerve section in adult and neonatal rats. J Comp Neurol 1989;284:215-30.

Honig MG, Hume RI. DiI and diO: versatile fluorescent dyes for neuronal labelling and pathway tracing. Trends Neurosci 1989;12:333-5, 340-1.

Innocenti GM, Clarke S, Kraftsik R. Interchange of callosal and association projections in the developing visual cortex. J Neurosci 1986;6:1384-409.

Keizer K, Kuypers HGJM, Huisman AM, Dann O. Diamidino Yellow Dihydrochloride (DY.2HCl); a new fluorescent retrograde neuronal tracer, which migrates only very slowly out of the cell. Exp Brain Res 1983;51:179-191.

Ljungberg C, Novikov L, Kellerth JO, Ebendal T, Wiberg W. The neurotrophins NGF and NT-3 reduce sensory neuronal loss in adult rat after peripheral nerve lesion. Neurosci Lett 1999;262:29-32.

Lowrie MB, Lavalette D, Davies CE. Time course of motoneurone death after neonatal sciatic nerve crush in the rat. Dev Neurosci 1994;16:279-84.

Lowrie MB, Vrbová G. Dependence of postnatal motoneurones on their targets: review and hypothesis. Trends Neurosci 1992;15:80-4.

Madison RD, Archibald SJ, Brushart TM. Reinnervation accuracy of the rat femoral nerve by motor and sensory axons. J Neurosci 1996;16:5698-703.

Martin LJ, Kaiser A, Price AC. Motor neuron degeneration after sciatic nerve avulsion in adult rats evolves with oxidative stress and is apoptosis. J Neurobiol 1999;40:185-201.

Mattson P, Meijer B, Svensson M. Extensive neuronal cell death following intracranial transection of the facial nerve in the adult rat. Brain Res Bull 1998;49:333-41.

Molander C, Aldskogius H. Directional specificity of regenerating neurons after peripheral nerve crush or transection and epineurial suture. A sequential double-labelling study in the rat. Restor Neurol Neurosci 1992;4:339-44.

Naumann T, Härtig W, Frotscher M. Retrograde tracing with Fluoro-Gold: different methods of tracer detection at the ultrastructural level and neurodegenerative changes of back-filled neurons in long-term studies. J Neurosci Methods 2000;103;11-21. 
Navarro X, Lázaro JJ, Butí M, Verdú E, Fábregas PJ, Castellanos B. Electrophysiological evaluation of spinal reflexes during epidural anesthesia in an experimental model. Muscle Nerve 1996;19:29-36.

Navarro X, Verdú E, Butí M. Comparison of regenerative and reinnervating capabilities of different functional types of nerve fibers. Exp Neurol 1994;129:217-24.

Novikova L, Novikov L, Kellerth O. Persistent neuronal labelling by retrograde fluorescent tracers: a comparison between Fast Blue, Fluoro-Gold and various dextran amines. J Neurosci Meth 1997;74:9-15.

Onifer SM, White LA, Whittemore SR, Holets VR. In vitro labeling strategies for identifying primary neural tissue and a neuronal cell line after transplantation in the CNS. Cell Transplantation 1993;2:131-49.

Peyronnard JM, Charron L, Lavoie J, Messier JP. Differences in Horseradish Peroxidase labelling of sensory, motor and sympathetic neurons following chronic axotomy of the rat sural nerve. Brain Res 1986;364:137-50.

Peyronnard JM, Charron L, Lavoie J, Messier JP, Bergouignan FX. A comparative study of the effects of chronic axotomy, crush lesion and re-anastomosis of the rat sural nerve on horseradish peroxidase labelling of primary sensory neurons. Brain Res 1988;443:295-309.

Pollin MM, McHanwell S, Slater CR. The effect of age on motor neurone death following axotomy in the mouse. Development 1991;112:83-9.

Puigdellívol-Sánchez A, Prats-Galino A. Ruano-Gil D, Molander C. Efficacy of the fluorescent dyes Fast Blue, Fluoro-Gold, and Diamidino Yellow for retrograde tracing to dorsal root ganglia after subcutaneous injection. J Neurosci Meth 1998;86:7-16.

Puigdellívol-Sánchez A, Prats-Galino A. Ruano-Gil D, Molander C. Fast Blue and Diamidino Yellow as retrograde tracers in peripheral nerves: efficacy of combined nerve injection and capsule application to transected nerves in the adult rat. J Neurosci Meth 2000;95:103-10.

Rende M, Granato A, Lo Monaco M, Zelano G, Toesca A. Accuracy of reinnervation by peripheral nerve axons regenerating across a 10-mm gap within an impermeable chamber. Exp Neurol 1991;111:332-9.

Schmued CL, Fallon JH. Fluoro-Gold: a new fluorescent retrograde axonal tracer with numerous unique properties. Brain Res 1986;377:147-154.

Snider WD, Elliott JL, Yan Q. Axotomy-induced neuronal death during development. J Neurobiol 1992;23:1231-46.

Snider WD, Thanedar S. Target dependence of hypoglossal motor neurons during development in maturity. J Comp Neurol 1989;279:489-98. 
St. John PA. Toxicity of "DiI" for embryonic rat motoneurons and sensory neurons in vitro. Life Sci 1991;49:2013-21.

Sterio DC. The unbiased estimation of number and sizes of arbitrary particles using the disector. $\mathbf{J}$ Microsc 1984;134:127-36.

Suzuki G, Ochi M, Shu N, Uchio Y, Matsuura Y. Sensory neurons regenerate more dominantly than motoneurons during the initial stage of the regenerating process after peripheral axotomy. Neuroreport 1998;9:3487-92.

Törnqvist E, Aldskogius H. Motoneuron survival is not affected by the proximo-distal level of axotomy but by the possibility of regenerating axons to gain access to the distal nerve stump. J Neurosci Res 1994;39:159-65.

Valero-Cabré A, Tsironis K, Skouras E, Perego G, Navarro X, Neiss WF. Superior muscle reinnervation after autologous nerve graft or poly-l-lactide- $\varepsilon$-caprolactone (PLC) tube implantation in comparison to silicone tube repair. J Neurosci Res 2001;63:214-23.

Vanden-Noven S, Wallace N, Muccio D, Turtz A, Pinter MJ. Adult spinal motoneurons remain viable despite prolonged absence of functional synaptic contact with muscle. Exp Neurol 1993;123:147-56.

Verdú E, Navarro X. Comparison of immunohistochemical and functional reinnervation of skin and muscle after peripheral nerve injury. Exp Neurol 1997;146:187-98.

Verestergaard S, Tandrup T, Jakobsen J. Effect of permanent axotomy on number and volume of dorsal root ganglion cell bodies. J Comp Neurol 1997;388:307-12.

Wada S, Yone K, Ishidou Y, Nagamine T, Nakahara S, Niiyama T, Sakou T. Apoptosis following spinal cord injury in rats and preventive effect of N-methyl-D-aspartate receptor antagonist. $\mathrm{J}$ Neurosurg (Spine 1) 1999;91:98-104.

Weiss ML, Cobbett P. Intravenous injection of Evans Blue labels magnocellular neuroendocrine cells of the rat supraoptic nucleus in situ after dissociation. Neuroscience 1992;48:383-95.

Ygge J. Neuronal loss in lumbar dorsal root ganglia after proximal compared to distal sciatic nerve resection: a quantitative study in the rat. Brain Res 1989;478:193-5.

Ygge J, Aldskogius H. Intercostal nerve transection and its effect on the dorsal root ganglion. A quantitative study on ganglion cell number and sizes. Exp Brain Res 1984;55:402-8.

Yu WA. Sex differences in neuronal loss induced by axotomy in the rat brain stem motor nuclei. Exp Neurol 1988;102:230-5. 
Table I. Experimental groups, number of subjects studied with morphological (n morph) and electrophysiological (n phys) techniques, nerve transected (or in case of the tibial, just injected), 1st tracer applied immediately after the sciatic transection ( 0 day) or saline injected into the tibial nerve without nerve transection, and 2nd tracer applied once completed the 90 days follow-up period.

Two animals, corresponding to the FG-DY and S-FG groups (bilateral experiments) died during the second tracer application, after the electrophysiological recordings, explaining why only 6 rats were analysed morphologically in each of these groups.

\begin{tabular}{llccccc}
\hline Group & Nerve & n phys & n morph & Vehicle & $\begin{array}{c}\text { 1st Tracer } \\
(0 \text { day })\end{array}$ & $\begin{array}{c}\text { 2nd Tracer } \\
\text { (90 days })\end{array}$ \\
\hline FB-DY & Right sciatic & 8 & 8 & Capsule & FB & DY \\
FG-DY & Right sciatic & 8 & 6 & Capsule & FG & DY \\
DY-FB & Right sciatic & 7 & 7 & Capsule & DY & FB \\
S-DY & Left sciatic & 8 & 8 & Capsule & Saline & DY \\
S-FG & Left sciatic & 8 & 6 & Capsule & Saline & FG \\
S-FB & Left sciatic & 7 & 7 & Capsule & Saline & FB \\
INJ & Right tibial & 8 & 8 & Injection & Saline & - \\
CONTR & Left tibial & 8 & 8 & - & - & - \\
\hline
\end{tabular}


Table II. Counted profiles in every tenth section in DRGs L4-L5 and in every fourth section of the lumbar spinal cord. Mean values and percentages without correction for split cells or cells lost by counting in only some of the sections.

1- Single labelling of regenerating neurones (application at 90 days): profiles labelled with FB, FG or DY, applied at the cut end of the sciatic nerve at the end of the regeneration period. 2-Labelling with the first tracer in double labelling experimentes: profiles single or double labelled with FB, FG or DY, applied at the cut end of the right sciatic nerve the first day of the experiment, immediately after section and before suture repair. 3-Labelling with the second tracer in double labelling experiments: DY labelled profiles, single or double labelled, in the groups originally labelled with FB or FG (FB-DY and FG-DY groups, respectively); FB profile counts in the group originally labelled with DY (DY-FB group). 4- Double labelling: The double labelled DRGs sensory neurones and spinal cord motoneurones shown as percentages of the total number of neurones containing the tracer applied to label the original population of the nerve in the FB-DY, FG-DY and DY-FB groups after 90 days. 5- Total number of counted profiles, single or multiple labelled, with any of the tracers in the FB-DY, FG-DY and DY-FB groups after 90 days. 
DRG Spinal cord DRG Spinal cord DRG Spinal cord SINGLE LABELLING (LEFT HINDLIMB)

\begin{tabular}{|c|c|c|c|c|c|c|}
\hline \multirow{3}{*}{$\begin{array}{l}\text { Groups } \\
\mathbf{1} \\
\text { Mean }\end{array}$} & \multicolumn{2}{|c|}{ S-FB } & \multicolumn{2}{|c|}{ S-FG } & \multicolumn{2}{|c|}{ S-DY } \\
\hline & \multicolumn{2}{|c|}{ FB } & \multicolumn{2}{|c|}{ FG } & \multicolumn{2}{|c|}{ DY } \\
\hline & 880.3 & 223.1 & 825.7 & 281.5 & 655.7 & $191.6^{\#}$ \\
\hline SD & 176.0 & 58.5 & 235.8 & 32.3 & 202.0 & 42.3 \\
\hline
\end{tabular}

DOUBLE LABELLING (RIGHT HINDLIMB)

\begin{tabular}{lccc} 
Groups & FB-DY & FG-DY & DY-FB \\
\hline $\mathbf{2}$ & Total FB & Total FG & Total DY \\
& $($ FB+FBDY $)$ & (FG+FGDY $)$ & $($ DY+DYFB $)$
\end{tabular}

\begin{tabular}{lcccccc} 
Mean & 885.9 & 328.9 & 788.0 & $124.3^{\# *}$ & 868.6 & 316.0 \\
SD & 430.5 & 102.8 & 157.8 & 29.1 & 150.4 & 48.5 \\
\hline $\mathbf{3}$ & Total DY & Total DY & Total FB \\
& (DY+FBDY) & (DY+FGDY) & (FB+DYFB)
\end{tabular}

\begin{tabular}{|c|c|c|c|c|c|c|}
\hline Mean & 558.9 & 91.3 & 558.8 & 106.0 & $924.4^{*}$ & $238.9^{\# *}$ \\
\hline SD & 436.8 & 117.1 & 238.7 & 59.0 & 177.4 & 54.1 \\
\hline$\overline{4}$ & \multicolumn{2}{|c|}{ FBDY/(FBDY+FB) } & \multicolumn{2}{|c|}{ FGDY/(FGDY+FG) } & \multicolumn{2}{|c|}{$\mathrm{DYFB} /(\mathrm{DYFB}+\mathrm{DY})$} \\
\hline Mean & $54.1 \%$ & $26.7 \%$ & $64.0 \%$ & $37.3 \%$ & $82.4 \%$ \#* & $66.8 \%$ \#* \\
\hline SD & $26.2 \%$ & $31.3 \%$ & $16.1 \%$ & $19.5 \%$ & $7.7 \%$ & $13.1 \%$ \\
\hline$\overline{5}$ & \multicolumn{2}{|c|}{$\mathrm{FB}+\mathrm{DY}+\mathrm{FBDY}$} & \multicolumn{2}{|c|}{ FG+DY+FGDY } & \multicolumn{2}{|c|}{$\mathrm{DY}+\mathrm{FB}+\mathrm{DYFB}$} \\
\hline Mean & 901.3 & 333.7 & 839.8 & $180.6^{\# *}$ & 1075.8 & 343.4 \\
\hline SD & 425.5 & 104.0 & 188.2 & 53.8 & 165.8 & 43.9 \\
\hline
\end{tabular}

1: $\mathrm{p}=0.001$ \# vs $\mathrm{S}-\mathrm{FG}$

2: $\mathrm{p}=0.001$ \#: vs FB-DY, $\mathrm{p}=0.0001 *$ : vs DY-FB

3: $\mathrm{p}<0.05$ \#: vs FB-DY, *: vs FG-DY

4: $\mathrm{p}<0.05$ \#: vs FB-DY, *: vs FG-DY

5: p<0.0001 \#: vs FB-DY, *: vs DY-FB 
Table III. Amplitude and latency of CMAPs recorded from medial gastrocnemious (GM), anterior tibialis (TA) and plantar (PL) muscles and of CNAPs from the digital nerves of the 2nd (2 dig.n), 4th (4 dig.n) and 5th (5 dig.n) toes in the different experimental groups evaluated. In three groups the right sciatic nerve was cut and exposed to FB, FG and DY (FB-DY, FG-DY and DY-FB groups) and the left sciatic nerve exposed to saline (S-FB, S-FG and S-DY groups), 90 days before evaluation. All the parameters obtained 90 days after nerve section in all the injured groups are significantly different from the controls (CONTR). 


\begin{tabular}{|c|c|c|c|c|c|c|c|}
\hline & FB-DY & FG-DY & DY-FB & S-FB & S-FG & S-DY & CONTR \\
\hline \multicolumn{8}{|c|}{ CMAP amplitude $(\mathrm{mV})$} \\
\hline GM m & $25.8 \pm 1.5$ & $26.4 \pm 1.4$ & $26.1 \pm 0.4$ & $28.4 \pm 1.2$ & $29.3 \pm 1.0$ & $26.0 \pm 0.5^{5}$ & $43.5 \pm 0.8$ \\
\hline TA m & $19.9 \pm 1.2$ & $24.4 \pm 1.3^{1}$ & $23.1 \pm 0.9$ & $18.6 \pm 0.9$ & $24.4 \pm 1.1^{4}$ & $23.3 \pm 1.0^{4}$ & $42.0 \pm 0.6$ \\
\hline PL m & $1.6 \pm 0.2$ & $1.6 \pm 0.2$ & $1.7 \pm 0.1$ & $1.5 \pm 0.1$ & $1.8 \pm 0.2$ & $1.7 \pm 0.1$ & $8.0 \pm 0.5$ \\
\hline \multicolumn{8}{|c|}{ CMAP latency (msec) } \\
\hline GM m & $1.80 \pm 0.14$ & $1.83 \pm 0.17$ & $1.41 \pm 0.03$ & $1.58 \pm 0.14$ & $1.51 \pm 0.09$ & $1.41 \pm 0.03$ & $1.28 \pm 0.04$ \\
\hline TA m & $1.81 \pm 0.05$ & $1.80 \pm 0.04$ & $1.92 \pm 0.06$ & $1.58 \pm 0.05^{1}$ & $1.64 \pm 0.05^{2}$ & $1.85 \pm 0.09^{3,4}$ & $1.34 \pm 0.03$ \\
\hline PL m & $3.75 \pm 0.10$ & $3.67 \pm 0.13$ & $3.85 \pm 0.08$ & $3.79 \pm 0.19$ & $3.52 \pm 0.08$ & $3.51 \pm 0.09^{3}$ & $2.50 \pm 0.08$ \\
\hline \multicolumn{8}{|c|}{ CNAP amplitude $(\mu V)$} \\
\hline 2dig. $n$ & $6.54 \pm 2.01$ & $6.65 \pm 1.66$ & $6.49 \pm 0.48$ & $5.50 \pm 1.45$ & $6.77 \pm 1.49$ & $4.35 \pm 1.27$ & $17.79 \pm 0.77$ \\
\hline 4dig. $n$ & $0.82 \pm 0.82$ & $6.25 \pm 1.43^{1}$ & $3.36 \pm 1.21$ & $5.50 \pm 1.73$ & $6.67 \pm 1.06$ & $4.66 \pm 1.32$ & $20.31 \pm 1.23$ \\
\hline 5dig. $n$ & $4.86 \pm 1.74$ & $6.67 \pm 1.06$ & $5.47 \pm 0.42$ & $5.46 \pm 1.77$ & $5.18 \pm 1.59$ & $4.27 \pm 1.16$ & $20.53 \pm 1.19$ \\
\hline \multicolumn{8}{|c|}{ CNAP latency (msec) } \\
\hline 2dig. $n$ & $3.77 \pm 0.14$ & $3.94 \pm 0.21$ & $3.75 \pm 0.13$ & $3.38 \pm 0.30$ & $3.44 \pm 0.08$ & $3.41 \pm 0.10$ & $1.75 \pm 0.03$ \\
\hline 4dig. $n$ & $4.44 \pm 0.00$ & $3.78 \pm 0.17$ & $4.25 \pm 0.29$ & $3.59 \pm 0.12$ & $3.77 \pm 0.16$ & $3.72 \pm 0.30$ & $1.83 \pm 0.05$ \\
\hline 5dig. $n$ & $3.41 \pm 0.11$ & $3.73 \pm 0.20$ & $3.66 \pm 0.19$ & $3.17 \pm 0.20$ & $3.48 \pm 0.14$ & $3.57 \pm 0.24$ & $1.75 \pm 0.04$ \\
\hline
\end{tabular}

p $<0.05{ }^{1}$ : vs FB-DY, 2 : vs FG-DY, ${ }^{3}$ : vs DY-FB, ${ }^{4}$ : vs S-FB, ${ }^{5}$ :vs S-FG 
Figure 1. Experimental design: on day 0 the sciatic nerve was sectioned bilaterally and one of three different tracers (FB in blue, DY in yellow and FG in reddish) were applied in each group on the right side, while saline was applied on the left side. The injured nerves were repaired by suture after thirty minutes. On day 90, DY was applied in the groups previously labelled by FB or FG, while the group previously labelled by DY received FB (right side). On the left side, the same tracers as those used day 0 on the left side were applied.

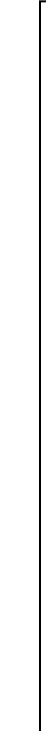

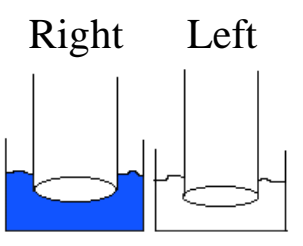
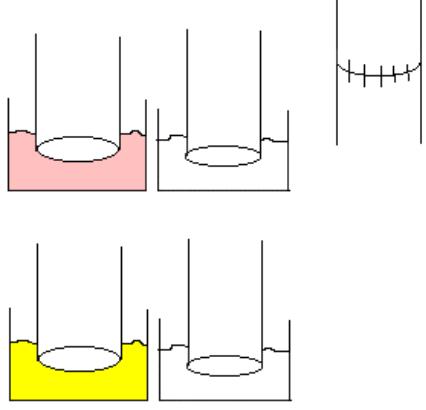

Day 0: Bilateral section

\section{- tracer \\ bilateral application \\ suture}

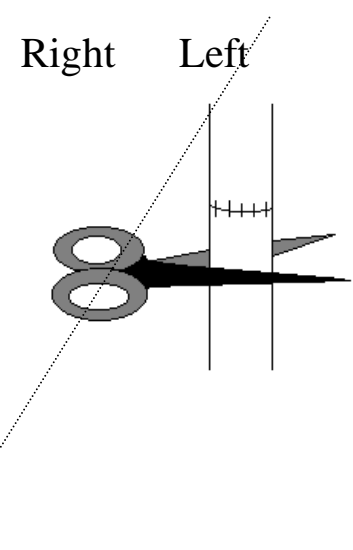

Day 90: Bilateral section
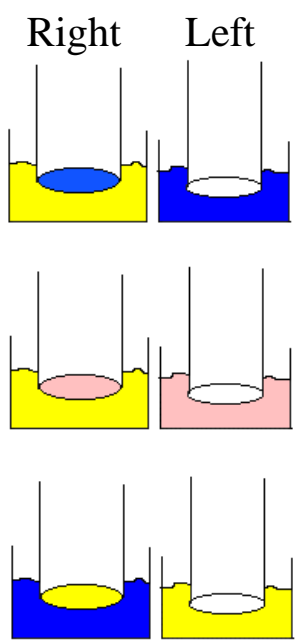

- tracer application
Formatat: Esquerra: 0,5 cm, Dreta: $0,5 \mathrm{~cm}$, Distància de la capçalera des de la vora: $1,25 \mathrm{~cm}$, Distància del peu

des de la vora: $1,25 \mathrm{~cm}$ 
Figure 2.

Formatat: Tipus de lletra: Times, No

A. Ultraviolet filter. Group of motoneurones single labelled with FG in the S-FG group.

B. Violet filter. Group of DRG cells single labelled with FB, DY or double labelled

-FBDY- in the DY-FB group. The DY accumulated in the cell bodies for months may appear as yellow granules evenly distributed in the cytoplasm (arrowheads) in some cells.

C. Violet filter. Group of DRG cells single labelled with DY in the S-DY group. When applied at the end of the regeneration period, the DY tends to concentrate at the periphery of the nucleus and less intensively in the cytoplasm, without forming granules.
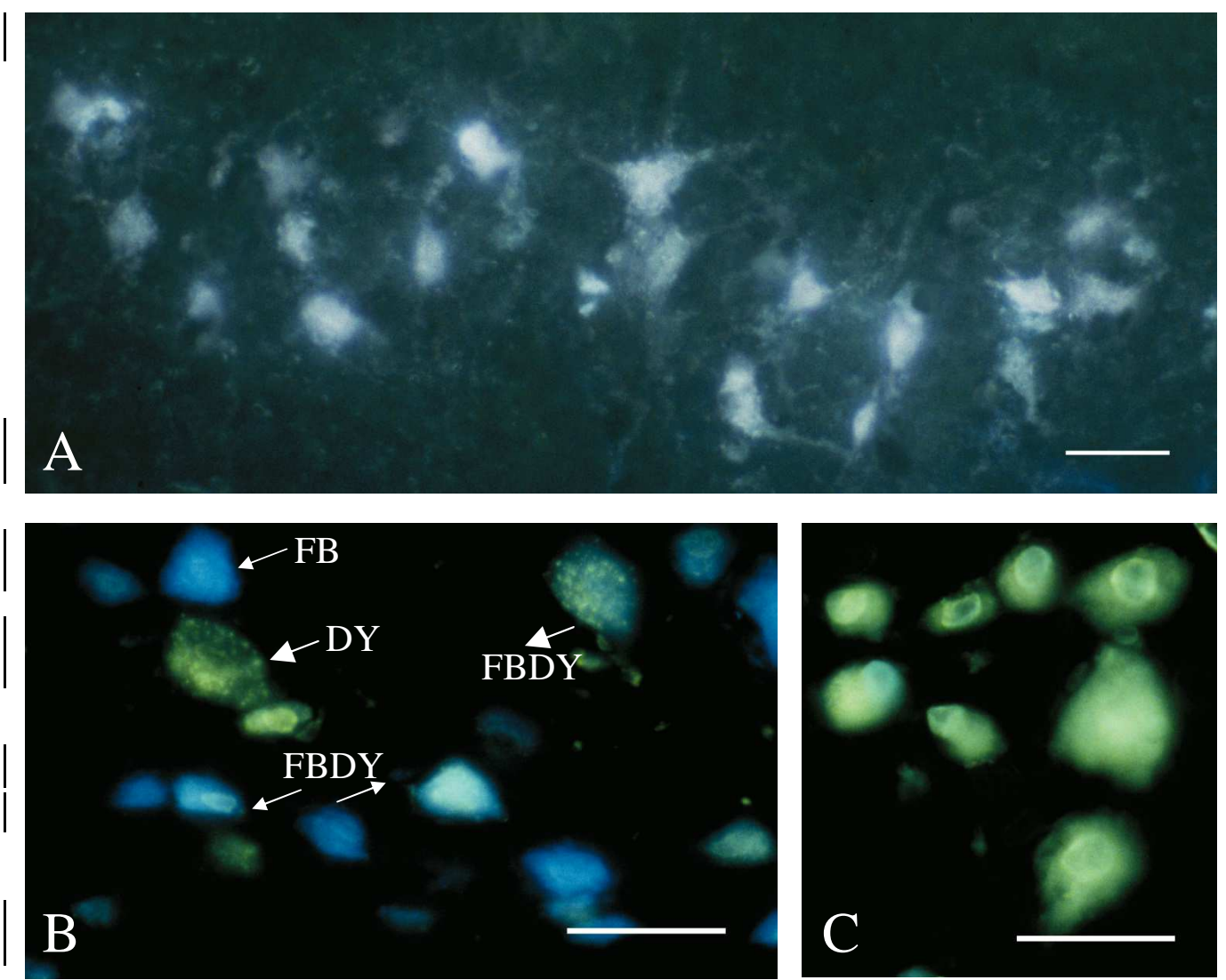
Figure 3. Number of cells single labelled by the first (1st tracer), the second tracer ( 2 nd tracer), and both tracers (double labelled) in each group. The first panel shows the DRG and the second the spinal cord.

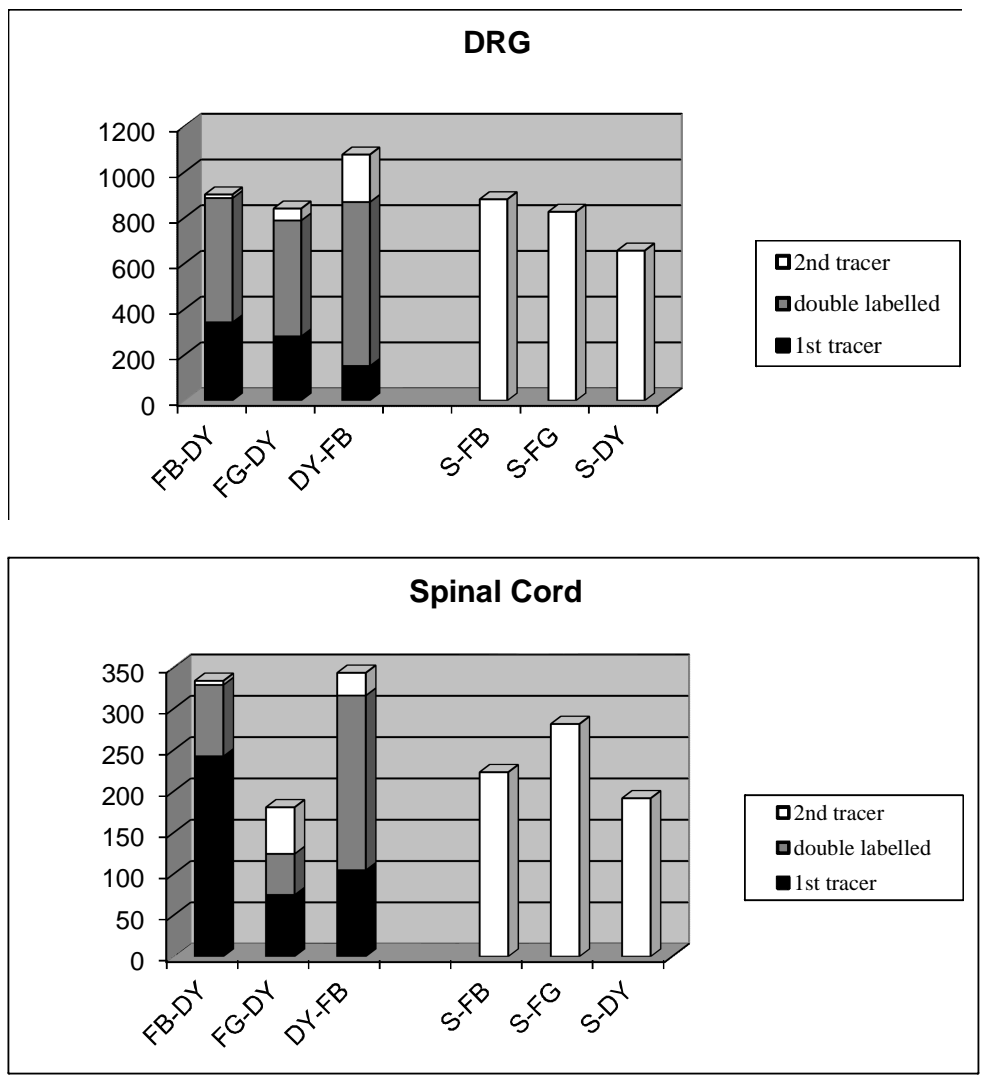

